\title{
ANEMIA FERROPRIVA / FERROPÊNICA EM GESTANTES: uma revisão integrativa de literatura
}

\author{
Sheila Viviany Lima de M. LOPES ${ }^{1}$ \\ Ieda Rodrigues de FREITAS ${ }^{2}$ \\ Maria da conceição C. MACIEL ${ }^{3}$
}

\begin{abstract}
${ }^{1}$ Enfermeira. Especialista em Saúde Coletiva Com Ênfase no Programa de Saúde da Família ( FAZER); PósGraduada Em Gestão do Trabalho e da Educação na Saúde (EAD) UFRN;Especialista em Gestão da Clínica No Sus -Regulação Em Saúde e Mestranda em Saúde coletiva e Gestão Hospitalar pela FACNORT. João Pessoa - PB.

${ }^{2}$ Bacharel em Letras-.Autarquia de Ensino Superior de Arco Verde Pernambuco- PE( AESA), Especialista Em Saúde Pública com Habilitação Sanitarista-Universidade de Ribeirão Preto (UNAERP) e Mestranda em Saúde coletiva e Gestão Hospitalar pela FACNORT. Caruaru-PE.

${ }^{3}$ Assistente social. Universidade católica de Pernambuco, Especialista em Saúde Pública- Universidade de Ribeirão Preto (UNAERP) e Mestranda em Saúde coletiva e Gestão Hospitalar pela FACNORT. Caruaru-PE.
\end{abstract}

Recebido em: 27/12/2014 - Aprovado em: 19/06/2015 - Disponibilizado em: 15/07/2015

RESUMO:A anemia ferropênica, apesar de ser uma das carências mais prevalentes no mundo, é um problema que ainda persiste, afetando, na maioria dos casos, gestantes e recem nascidos incluindo o elevado risco de mortalidade materna durante o período perinatal, o baixo peso ao nascer e partos pré-termos. Diante deste o estudo tem o objetivo geral buscar e avaliar as evidências disponíveis na literatura, a prevalência da anemia ferropriva / ferropênica em gestantes e os fatores relacionados, bem como as conseqüências para o recém-nascido; Trata-se de uma revisão Integrativa que utilizou artigos científicos e publicações de órgãos governamentais disponíveis nas bases de dados da Scielo, Bireme Lilacs e Google acadêmico para embasamento teórico. Como resultado, constatou-se que a falta de sse micronutriente pode causar grandes problemas relacionados à saúde materna e infantil como: anemia ferropriva, subnutrição infantil, e anemia megaloblástica. Logo, percebe-se que o uso desse micronutriente é essencial para um bom pré-natal e para garantir o bem estar materno e fetal. Assim, políticas públicas que venham a incentivar ainda mais a suplementação materna dessas substâncias são de grande valia, uma vez que reduzem a morbi-mortalidade materna e neonatal.

Palavras-chave:Anemia Ferropriva. Anemia em Gestantes. Anemia Carêncial.

SUMMARY: Iron deficiency anemia, despite being one of the most prevalent deficiencies in the world, is a problem that still persists, affecting, in most cases, pregnant women and newborn infants including the high risk of maternal mortality during the perinatal period, low weight at birth and preterm births. Before this study has the general objective search and evaluate evidence available in the literature, the prevalence of iron deficiency anemia / iron deficiency in pregnant women and related factors as well as the consequences for the newborn; This is an integrative review using scientific articles and publications from government agencies available in SciELO databases, Bireme Lilacs and Google Scholar for theoretical basis. As a result, it was found that the lack of micronutrient iff can cause major problems related to maternal and child health as iron deficiency anemia, child malnutrition, and megaloblastic anemia. Therefore, one can see that the use of this nutrient is essential for prenatal good and to ensure maternal and fetal well-being. Thus, public policies that will further encourage maternal supplementation of these substances are of great value, since they reduce maternal and neonatal morbidity and mortality.

Keywords: Iron Deficiency Anemia. Anemia in pregnant women. Nutritional anemia. 


\section{INTRODUÇÃO}

${ }^{2,5}$ Segundo o autor relata que em nossa prática temos observado que o problema da anemia ferropriva / ferropênica pode causar inúmeras alterações de saúde na população envolvida por essa carência. É conhecida, a partir da literatura especializada, a grande prevalência da anemia por carência de ferro em todas as partes do mundo, notadamente nas regiões mais pobres, o que torna seu estudo, tratamento e prevenção de grande importância em termos de pré-requisito para melhoria da saúde pública.

${ }^{1} \mathrm{O}$ desenvolvimento gestacional fisiológico está associado a ajustes que acarretam acentuadas mudanças no organismo materno, incluindo aí a composição dos elementos figurados e humorais no sangue circulante. Provavelmente, em nenhuma outra fase do ciclo vital exista maior mudança no funcionamento e forma do corpo humano em tão curto espaço de tempo, entretanto ${ }^{6}$ muitas dessas mudanças iniciam-se desde o momento da nidação e se estendem por todo período gestacional, até o término da lactação. $\mathrm{O}$ conhecimento de tais mudanças é necessária para uma adequada avaliação do "processo saúde / doença" da gestante e as sequelas para o RN.
Dentre os órgãos mais afetados por mudanças durante o período gestacional está o útero que sofre modificações de hipertrofia e dilatação, requerendo um aumento da vascularização pela necessidade de maior perfusão sanguínea. A placenta, aí implantada, devido ao aumento progressivo, passa a necessitar de um incremento correlato do fluxo sanguíneouteroplacentário com a evolução da gestação, o que demanda, também, um aumento do número de vasos sanguíneos ${ }^{1}$.

${ }^{1}$ Como critérios para definir a condição de anemia em gestantes os valores de hemoglobina menores que $11 \mathrm{mg} / \mathrm{dl}$. e VCM inferior $85 \mathrm{dl}$

${ }^{1,6}$ Atualmente a deficiência do mineral ferro é reconhecida como o mais comum estado de déficit humano, das mulheres em idade fértil 10 a $30 \%$ podem apresentar sinais de deficiência de ferro, enquanto que durante o período gestacional este número sobe para 10 a $60 \%$. Dentre as grávidas e lactentes, no primeiro ano de vida, 20 a $60 \%$ chegam a apresentar anemia ferro deficitária. Além de sua elevada magnitude, a anemia ferropriva / ferropênica possui marcante implicação no processo saúde-doença, interagindo com outras doenças carenciais e não-carenciais.

Nessa temática - anemia ferropriva / ferropênica em gestantes - tanto em nível de 
teoria quanto de prática, é relevante avançar na idéia de que a carência de ferro promove conseqüências negativas tanto para a mulher como para o recém-nascido. Partindo desta premissa podemos citar patologias que podem surgir com a gravidez ou já estarem instaladas antes que ela ocorra, tais como: aborto espontâneo, partos prematuros, hemorragias durante o trabalho de parto, hipóxia, RN de baixo peso, anemia para o $\mathrm{RN}$, baixa na imunidade.

Essa condição é responsável pela diminuição nas chances de sobrevivência dos recém-nascidos e aumento do risco de apresentar seqüelas mais graves e em maior número que outras crianças nascidas de mães sem a deficiência do mineral ferro.

A abordagem deste tema se justifica pelo fato de pretendermos fundamentar, a prevalência da anemia ferropriva / ferropênica, os fatores relacionados, e as conseqüências para o binômio mãe-filho, bem como fortalecer as orientações durante o pré-natal acerca da adequada prevenção da anemia ferropriva / ferropênica.

Esse levantamento bibliográfico irá contribuir para desenvolver trabalhos de orientação alimentar com a população de gestantes visando prevenção e tratamento dos sinais e sintomas de anemia por deficiência de ferro, na tentativa de mudanças nos hábitos alimentares da população.

Apesar dos avanços tecnológicos aplicados à assistência ao pré-natal nos últimos anos, as taxas de anemia ferropriva / ferropênica têm se mostrado elevadas. Daí a necessidade de estudos que venham esclarecer as causas que levam a estes índices e qual a sua implicação para as mulheres e os recém-nascidos.

A pesquisa teve como objetivo geral buscar e avaliar as evidências disponíveis na literatura, a prevalência da anemia ferropriva / ferropênica em gestantes e os fatores relacionados, bem como as conseqüências para o recém-nascido.

\section{MATERIAIS E MÉTODOS}

Para a elaboração da presente revisão integrativa as seguintes etapas foram percorridas: definição da questão norteadora (problema) e objetivos da pesquisa;estabelecimento de critérios de inclusão e exclusão das publicações (seleção da amostra); busca na literatura; análise e categorização dos estudos, apresentação e discussão dos resultados.

Para guiar a pesquisa, formulou-se a seguinte questão: $O$ que foi produzido na literatura sobre a prevalência da anemia ferropriva / ferropênica em gestantes e os fatores relacionados, como as conseqüências para o recém-nascido. 
Realizou-se em setembro de 2014 a busca daspublicações indexadas nas seguintes bases de dados: Base de Dados LILACS (Literatura Latino Americana e do Caribe em Ciências da Saúde), e na biblioteca SciELO (Scientific Electronic Library on Line) com os seguintes critérios de inclusão: estudos que abordem a temática Anemia Ferropriva em gestantes, indexados nas base de dados, publicados no período1987 a 2009, resultante de pesquisa primaria qualitativas, quantitativas e estudo teóricos e com acesso on-line em texto completo em idioma português. Foram utilizados os seguintes descritores controlados: anemia and ferropriva and gestantes.

Como critérios de exclusão adotados foram: artigos científicos sem o acesso online, escritos em outro dioma que não os selecionados para o estudo, que não possuíam acesso online em texto completo e que não correspondia a questão norteadora.

Nas busca dos artigos nas bases de dados encontrou-se: 57 artigos na LILACS, 21 artigos na SciELO.

Ao se aplicar o critério de inclusão na leitura de título e resumo, foram selecionados nas bases de dados o seguinte quantitativo: LILACS - 24, SciELO - 14. Assim se obteve uma população de 38 artigos científicos. Posteriormente realizou- se a leitura na íntegra desta população, com objetivo de se refinar as informações em atenção a questão norteadora do estudo, neste processo foram excluídos 31 artigos sendo selecionado para a amostra deste estudo 7 artigos científicos.

$\mathrm{Na}$ perspectiva de sumarizar e organizar as informações utilizou-se o instrumentoque identifica a publicação com título do artigo, ano de publicação, autores, o que foi estudado, resultados, recomendações/conclusões juntamente com os critérios de avaliação de estudos de anemia ferropriva em gestantes e os fatores relacionados, bem como as consequiências para o recém-nascido. Os dados foram categorizados e discutidos segundo os objetivos da revisão integrativa.

\section{RESUTADOS E DISCUSSÕES}

Tabela 1 - Apresentação da síntese de artigos incluídos na revisão integrativa 


\begin{tabular}{|c|c|c|c|c|c|}
\hline TITULO DO ARTIGO & $\begin{array}{l}\text { ANO DE } \\
\text { PUBLICAÇ } \\
\text { ÃO }\end{array}$ & AUTOR & O QUE FOI ESTUDADO & RESULTADOS & RECOMENDA-ÇÕES/ CONCLUSÕES \\
\hline $\begin{array}{l}\text { Baixa escolaridade como fator } \\
\text { limitante para o combate à } \\
\text { anemia entre gestantes }\end{array}$ & 2006 & $\begin{array}{l}\text { VITOLO, Márcia } \\
\text { Regina; } \\
\text { BOSCAINI, } \\
\text { Camileand } \\
\text { BORTOLINI, } \\
\text { Gisele Ane. }\end{array}$ & $\begin{array}{l}\text { O impacto e a adesão de } \\
\text { uma intervenção baseada na } \\
\text { prescrição de suplemento de } \\
\text { ferro e orientações } \\
\text { alimentares nos níveis de } \\
\text { hemoglobina de gestantes de } \\
\text { baixa escolaridade de uma } \\
\text { Unidade Básica de Saúde. }\end{array}$ & $\begin{array}{l}\text { O uso do suplemento de ferro } \\
\text { foi referido por } 65 \% \text { das } \\
\text { gestantes do grupo intervenção, } \\
\text { sendo que } 67,7 \% \text { interromperam } \\
\text { o uso em algum momento. Os } \\
\text { motivos principais foram: } \\
\text { esquecimento }(43,2 \%) \text { e enjoo } \\
\text { e/ou vômito }(27,2 \%) \text {. Gestantes } \\
\text { com escolaridade inferior a } 8 \\
\text { anos de estudo apresentaram } 3 \\
\text { vezes mais risco na ocorrência } \\
\text { de anemia no terceiro trimestre. }\end{array}$ & $\begin{array}{l}\text { O uso de sulfato ferroso não se mostrou } \\
\text { associado à menor prevalência de anemia } \\
\text { Os resultados sugerem que são necessárias } \\
\text { mudanças estruturais nas condições } \\
\text { socioeconômicas para se modificar o o } \\
\text { quadro atual quanto à anemia ferropriva. }\end{array}$ \\
\hline $\begin{array}{l}\text { Estado nutricional e anemia } \\
\text { ferropriva em gestantes: } \\
\text { relação com o peso da criança } \\
\text { ao nascer. }\end{array}$ & 2005 & $\begin{array}{l}\text { ROCHA, Daniela } \\
\text { da Silva et al. }\end{array}$ & $\begin{array}{l}\text { O peso ao nascer reflete a } \\
\text { qualidade da atenção dada à } \\
\text { gestante durante esse } \\
\text { período de grande } \\
\text { vulnerabilidade, devendo } \\
\text { haver preocupação com o } \\
\text { seu estado nutricional, antes } \\
\text { e durante a gestação, e } \\
\text { observar os fatores de risco } \\
\text { associados. }\end{array}$ & $\begin{array}{l}\text { A maioria das gestantes } \\
\text { apresentou ganho de peso } \\
\text { inadequado durante a gestação. } \\
\text { A prevalência total de anemia } \\
\text { ferropriva foi de } 21,4 \% \text {, sendo } \\
\text { que essa aumentou com a idade } \\
\text { gestacional. A frequência de } \\
\text { baixo peso e peso insuficiente } \\
\text { ao nascer foi de } 8,9 \% \text { e } 28,6 \% \text {, } \\
\text { respectivamente. }\end{array}$ & $\begin{array}{l}\text { A avaliação do estado nutricional das } \\
\text { gestantes de baixo nível socioeconômico } \\
\text { identificou uma alta prevalência de } \\
\text { gestantes com estado nutricional } \\
\text { desfavorável, pois foram encontradas, } \\
\text { aproximadamente } 43,0 \% \text { de gestantes que } \\
\text { iniciaram o pré-natal com baixo peso, } \\
\text { sobrepeso e/ou obesidade. Ao correlacionar } \\
\text { as variáveis antropométricas das gestantes } \\
\text { com o peso ao nascer, observou-se que } \\
\text { todas as variáveis antropométricas } \\
\text { apresentaram relação estatisticamente } \\
\text { significante com o peso ao nascer, sendo } \\
\text { que o ganho de peso gestacional apresentou } \\
\text { maior correlacãa. }\end{array}$ \\
\hline
\end{tabular}




\begin{tabular}{|c|c|c|c|c|c|}
\hline 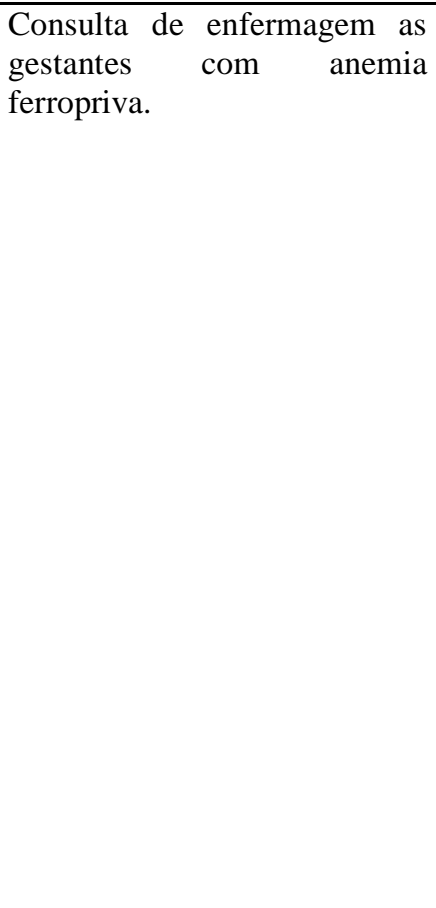 & 1999 & $\begin{array}{lr}\text { BARROS, } & \text { Sonia } \\
\text { Maria } & \text { Oliveira } \\
\text { and. } & \text { COSTA, } \\
\text { Cláudia } & \text { Aparecida } \\
\text { Ribeiro. } & \end{array}$ & $\begin{array}{l}\text { A Construção de um } \\
\text { protocolo de levantamento } \\
\text { de dados e diagnósticos de } \\
\text { enfermagem, e conhecer os } \\
\text { diagnósticos de enfermagem } \\
\text { mais frequentem. }\end{array}$ & $\begin{array}{l}\text { O protocolo de levantamento de } \\
\text { dados e diagnósticos de } \\
\text { enfermagem foi utilizado em } 52 \\
\text { consultas de enfermagem às } \\
\text { gestantes com anemia } \\
\text { ferropriva, e os diagnósticos de } \\
\text { enfermagem mais frequentem } \\
\text { foram: * Nutrição alterada: } \\
\text { ingesta menor que as } \\
\text { necessidades corporais } \\
\text { relacionada à verbalização de } \\
\text { falta de alimentos; Risco para } \\
\text { infecção relacionado à defesa } \\
\text { secundária insuficiente e pela } \\
\text { diminuição da hemoglobina;. } \\
\text { Manutenção do lar prejudicada } \\
\text { relacionada à falta de recursos } \\
\text { financeiros suficientes; Déficit } \\
\text { de conhecimento sobre a } \\
\text { alimentação adequada durante a } \\
\text { gestação; Risco para lesão fetal } \\
\text { relacionado à diminuição da } \\
\text { perfusão útero placentária. }\end{array}$ & $\begin{array}{l}\text { Na consulta de enfermagem às gestantes } \\
\text { com anemia ferropriva, o protocolo de } \\
\text { levantamento de dados e diagnósticos de } \\
\text { enfermagem mostrou-se um instrumento } \\
\text { eficaz, facilitou o relacionamento entre a } \\
\text { enfermeira e a gestante, a identificação, } \\
\text { controle e prevenção da anemia, e } \\
\text { proporcionou o planejamento das } \\
\text { intervenções de enfermagem } \\
\text { individualizadas. }\end{array}$ \\
\hline $\begin{array}{l}\text { Intervenções Nutricionais na } \\
\text { Anemia Ferropriva. }\end{array}$ & 1994 & $\begin{array}{l}\text { CARDOSO, Marly } \\
\text { A. and } \\
\text { PENTEADO, } \\
\text { Marilene de V. C. }\end{array}$ & $\begin{array}{l}\text { A necessidade } \\
\text { intervenções para o controle } \\
\text { da prevalência da anemia } \\
\text { por deficiência de ferro deve } \\
\text { ser determinada pela } \\
\text { magnitude da deficiência } \\
\text { nutricional e r pelo } \\
\text { conhecimento de seus } \\
\text { efeitos na qualidade de vida. }\end{array}$ & $\begin{array}{l}\text { O tratamento com ferro } \\
\text { medicamentoso deve ser } \\
\text { utilizado em todos os pacientes } \\
\text { com diagnóstico clínico } \\
\text { laboratorial de anemia, uma vez } \\
\text { que as modificações da dieta por } \\
\text { si só não podem corrigir a ADF. }\end{array}$ & $\begin{array}{l}\text { O abastecimento inadequado dos } \\
\text { suplementos de ferro e a baixa cobertura } \\
\text { dos serviços desaúde são as maiores } \\
\text { dificuldades encontradasem muitos } \\
\text { programas de suplementação comferro. }\end{array}$ \\
\hline $\begin{array}{llr}\text { Anemia e } & \text { Ferropenia } & \text { em } \\
\text { gestantes: } & \text { dissensos } & \text { de } \\
\text { resultados de um estudo } \\
\text { transversal. }\end{array}$ & 2007 & $\begin{array}{l}\text { BRESANI, } \\
\text { Cristiane } \\
\text { Campello; } \\
\text { SOUZA, Bresani } \\
\text { Ariani Impieri de; } \\
\text { BATISTA FILHO, }\end{array}$ & $\begin{array}{l}\text { Investigar se os baixos } \\
\text { níveis de } \mathrm{Hb} \text { correspondem } \\
\text { de fato à condição de anemia } \\
\text { ferropriva na gravidez. }\end{array}$ & $\begin{array}{l}\text { Considerou o ponto de corte de } \\
\mathrm{Hb}<11 \mathrm{~g} / \mathrm{dL} \text {, que das } 318 \\
\text { mulheres incluídas no estudo, } \\
7,6 \% \text { tinham ferropenia, sendo } \\
6,9 \% \text { não anêmicas e } 10,7 \% \\
\text { anêmicas. Do total das }\end{array}$ & $\begin{array}{l}\text { A freqüência de anemia não se comportou } \\
\text { como um proxi de anemia ferropriva, assim } \\
\text { como a frequêencia de ferropenia não } \\
\text { acompanhou na proporção esperada a de } \\
\text { anemia ferropriva, independente do ponto } \\
\text { de corte utilizado para a hemoglobina. }\end{array}$ \\
\hline
\end{tabular}




\begin{tabular}{|c|c|c|c|c|c|}
\hline & & $\begin{array}{l}\text { Malaquias and } \\
\text { FIGUEIROA, José }\end{array}$ & & $\begin{array}{lr}\text { participantes, 56,6\% } & \text { eram } \\
\text { anêmicas, } & \text { sendo } \\
\text { 45,9\%representados } & \text { por } \\
\text { anêmicas sem ferropenia. } & \\
\end{array}$ & $\begin{array}{l}\text { Ademais, ser anêmica apontou para uma } \\
\text { menor possibilidade de ferropenia. }\end{array}$ \\
\hline 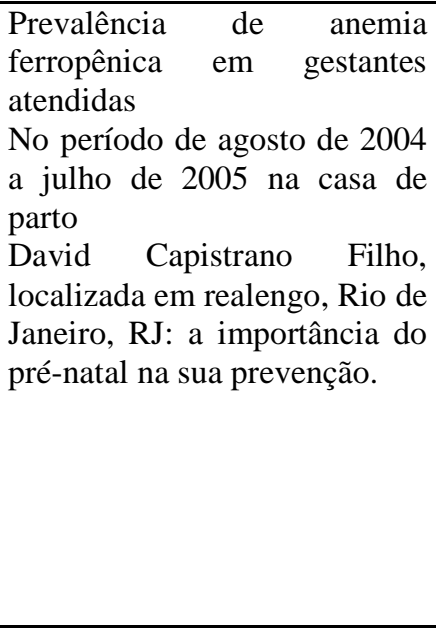 & 2005 & $\begin{array}{l}\text { VIANNA, A. C. } \\
\text { C.; Castro, D. L. } \\
\text { de; COELHO, R. } \\
\text { S. R }\end{array}$ & $\begin{array}{lr}\text { Prevenir } & a \\
\text { anemiaferropênica nessa } \\
\text { fase do ciclobiológico } \\
\text { gestacional. }\end{array}$ & $\begin{array}{l}\text { Com base nos dados obtidos, } \\
\text { concluiu-se que as gestantes } \\
\text { iniciaram tardiamente o } \\
\text { acompanhamento pré-natal; o } \\
\text { grau de escolaridade das } \\
\text { gestantes não interferiu } \\
\text { significativamente na renda } \\
\text { mensal monetária per capta; } \\
\text { todas as gestantes fizeram uso } \\
\text { diário da suplementação } \\
\text { medicamentosa com sulfato } \\
\text { ferroso, sendo que a maioria } \\
\text { ( } 73 \% \text { )ingeriu uma dose diária } \\
(30 \text { a } 60 \mathrm{mg}) \text { e } 27 \% \text { fizeram uso } \\
\text { de duas doses por dia ( } 60 \text { a } \\
120 \mathrm{mg}) \text {; }\end{array}$ & $\begin{array}{l}\text { Reslizar busa ativa das gestantes para } \\
\text { realização precose do pré natal e montar } \\
\text { um grupo de promoção da saúde. }\end{array}$ \\
\hline $\begin{array}{lll}\text { Prevalência } & \text { de } & \text { anemia } \\
\text { ferropriva em } & \text { gestantes, em } \\
\text { uma unidade de saúde da } & \text { de } \\
\text { família no município de } \\
\text { Panelas/PE. }\end{array}$ & 2010 & $\begin{array}{l}\text { Silva, Camilla } \\
\text { Thayran Neves } \\
\text { Silva, Fernanda } \\
\text { Gomes Enéas. }\end{array}$ & $\begin{array}{l}\text { Averiguar a prevalência de } \\
\text { anemia em gestantes tendo } \\
\text { em vista que a anemia é uma } \\
\text { patologia que interfere } \\
\text { diretamente na saúde da } \\
\text { gestante. }\end{array}$ & $\begin{array}{l}\text { A prevalência de anemia } \\
\text { encontrada entre as gestantes no } \\
\text { PSF de Cruzes do município de } \\
\text { Panelas/PE foi de } 12,22 \% \text {, } \\
\text { sendo classificada acima de } \\
4,7 \% \text { que é preconizado pelo } \\
\text { MS. Portanto cabe a enfermeira } \\
\text { deste PSF adotar medidas sócio- } \\
\text { educativas para que esses } \\
\text { números reduzam ainda mais, } \\
\text { garantido melhor qualidade de } \\
\text { vida para as gestantes desta } \\
\text { comunidade. }\end{array}$ & $\begin{array}{l}\text { A enfermagem deve fazer um } \\
\text { acompanhamento das gestantes, incentivar } \\
\text { a uma alimentação saudável, e ao uso } \\
\text { correto da suplementação de ferro. }\end{array}$ \\
\hline
\end{tabular}


${ }^{7}$ Por sua vez, foram avaliadas 168 gestantes de baixo nível socioeconômico e baixa escolaridade. Encontraram-se 41,3\% de gestantes com estado nutricional prégestacional inadequado, sendo $25,7 \%$ com baixo peso e 17,4\% com sobrepeso ou obesidade. A maioria das gestantes apresentou ganho de peso inadequado durante a gestação. Onde a prevalência total de anemia ferropriva foi de $21,4 \%$, sendo que esse aumentou com a idade gestacional.

${ }^{2}$ Todavia, este traz conhecimentos gerais sobre a anemia ferropriva e suas particularidades relacionadas à gestante assim como a realização de construção de um protocolo de levantamento de dados e diagnóstico de enfermagem a ser utilizado na consulta de enfermagem a gestantes com anemia ferropriva identificando os diagnosticos e propondo intervenções.

${ }^{4}$ Portanto este estudo expõe sobre o fornecimento de ferro suplementar a gestantes, nutrizes e lactentes em programas de assistência primária à saúde. Apesar da eficácia comprovada desses programas, sua eficiência é às vezes muito baixa, onde as dificuldades relacionadas ao abastecimento, distribuição e consumo dos suplementos de ferro são identificadas na grande maioria dos programas da rede básica de saúde.

${ }^{3} \mathrm{O}$ estudo descreve a frequencia da anemia ferropriva em gestantes de baixo risco , um estudo transversal com 318 gestantes atendidas no Instituto Materno Infantil Prof. Fernando Figueira-IMIP , em Recife, onde, Determinou-se a freqüência de anemia e/ou ferropenia em função da ferritina e dois diferentes pontos de corte ,portantoa freqüência de anemia foi reduzida para $37,4 \%$ e de anemia ferropriva para $7,9 \%$.

${ }^{10}$ Sem perder o eixo, observa-se que as gestantes iniciaram tardiamente $\mathrm{o}$ acompanhamento pré-natal; o grau de escolaridade das gestantes não interferiu significativamente na renda mensal monetária per capta; todas as gestantes fizeram uso diário da suplementação medicamentosa com sulfato ferroso, sendo que a prevalência de anemia identificada pela concentração de hemoglobina inferior a $11,0 \mathrm{mg} / \mathrm{dL}$ ocorreu em $23 \%$ das gestantes estudadas; a população anêmica desenvolveu anemia de grau leve.

${ }^{8}$ Dessa forma, afirma que a prevalência de anemia encontrada entre as gestantes no PSF de Cruzes do município de Panelas/PE foi de $12,22 \%$, sendo classificada acima de $4,7 \%$ que é preconizado pelo MS. Portanto a renda dessas mulheres pode ter interferido, de alguma forma, no desenvolvimento da anemia. E o não uso de sal de ferro de forma preventiva pode ter interferido 
significativamente no desenvolvimento da anemia. Além de todos esses fatores, é importante ressaltar que a enfermagem contribuiu e contribui para que a prevalência de anemia ferropriva na gestação diminua de maneira significativa. Para isso, a enfermagem faz acompanhamento das gestantes, incentiva a uma alimentação saudável, e ao uso correto da suplementação de ferro.

\section{CONSIDERAÇÕES FINAIS}

Diante do exposto ao longo do estudo, percebe-se que o uso do sulfato ferroso, mesmo em mulheres que não tenham anemia (profilático) é inevitável para que diminuam as chances de anemias, hemorragias pós-parto, hipertrofia placentária, mal estar e subnutrição em feto, que pode aumentar a morbi-mortalidade tanto materna quando fetal.

Pudemos identificar os riscos maternos e fetal com a falta de sulfato ferroso que são inúmeros, gerando consequências graves pra saúde materna e neonatal, transformando este um assunto de repercussão universal pela magnitude das repercussões.

A anemia ferropriva apesar de ser uma das carências mais prevalentes no mundo, é um problema que ainda persiste, afetando, na maioria dos casos, gestantes e crianças em idade pré-escolar gerando grande impacto na vida do ser humano quando analisados pelos os índices apresentados nas bibliografias estudadas, consequentemente apresentam um problema à Saúde Publica. (são consideráveis e que a enfermagem pode intervir de modo positivo na redução da taxa de anemia ferropriva / ferropênica e em suas consequiências para o recém nascido, com uma conscientização das grávidas sobre as causas que podem desencadear o processo de anemia ferropriva / ferropênica, assim como as intervenções necessárias.)

A partir desta pesquisa é possível acompanhar a evolução da opinião dos autores acerca dos processos que levam ao quadro de anemia por deficiência do mineral ferro bem como o conhecimento das gestantes acerca do processo que desencadeia a anemia ferropriva / ferropênica. Fica evidente que cada vez mais é necessário investir em pesquisas sobre como possa intervir nos atendimentos as gestantes visando à prevenção da anemia durante o pré-natal.

\section{REFERÊNCIA BIBLIOGRÁFICA}

1. Brasil. Ministério da Saúde. Manual técnico pré-natal e puerpério - Atenção Qualificada e Humanizada. Secretaria de atenção à saúde - Brasília: Ministério da Saúde; 2010. 
2.BARROS, S. M. O.; and COSTA, C. A.R..Consulta de enfermagem às gestantes com anemia ferropriva. Rev. LatinoAm.Enfermagem [online]. 1999, vol.7, n.4, pp. 105-111. ISSN 0104-1169.

3. BRESANI, C. C.; SOUZA, B. A. I.B. F.; Malaquiasand FIGUEIROA, J.N..Anemia e ferropenia em gestantes: dissensos de resultados de um estudo transversal. Rev. Bras. Saude Mater. Infant. [online]. 2007, vol.7, suppl.1, pp. s15-s21. ISSN 1519-3829. 4. CARDOSO, M. A.; and PENTEADO, M. V. C.. Intervenções nutricionais na anemia ferropriva. Cad. Saúde Pública [online]. 1994, vol.10, n.2, pp. 231-240. ISSN 0102$311 X$.

5. COSTA,R.S.S.. Carmo MGT, Jesus EFO. Níveis de ferro, cobre e zinco em colostro de puérperas adultas de recém--nascidos a termo e pré-termo e sua associação com as variáveis maternas e socioeconômicas. Rev Bras Saúde Mater Infant. 2002; 48(2):43-50.

6. CUNNINGHAME. Distúrbios hematológicos. In Complicações Médicas na Gestação / William M. Barron e Marshall D. Lindheimer; Trad. Walkiria M.F.Settineri. $2^{\mathrm{a}}$ ed. Porto Alegre: Artes Médicas 1996
7. ROCHA, D. S.; et al. Estado nutricional e anemia ferropriva em gestantes: relação com o peso da criança ao nascer. Rev. Nutr. [online]. 2005, vol.18, n.4, pp. 481489. ISSN 1415-5273.

8. Silva, C. T. N. S.. Prevalência de anemia ferropriva em gestantes, em uma anuidade de saúde da família no município de Panelas-PE / Trabalho de Conclusão de Curso (Enfermagem) -Faculdade do Vale do Ipojuca. -- Caruaru: FAVIP, $2010.22 \mathrm{f}$.

9. VITOLO, M. R.; BOSCAINI, C. and BORTOLINI, G. A. Baixa escolaridade como fator limitante para o combate à anemia entre gestantes. Rev. Bras. Ginecol. Obstet. [online]. 2006, vol.28, n.6, pp. 331339. ISSN 0100-7203.

10.VIANNA, A. C. C.; Castro, D. L. de; COELHO, R. S. R.. Prevalência de anemia ferropênica em gestantes atendidas no período de agosto de 2004 a julho de 2005 na Casa de Parto David Capistrano Filho, localizada em Realengo, Rio de Janeiro, RJ: a importância do pré-natal na sua prevenção.. 2005. Trabalho de Conclusão de Curso (Graduação em Licenciatura e Bacharelado em Ciências Biológicas) Universidade Castelo Branco. 